\title{
Frequency Domain Iterative Feedforward/Feedback Tuning for MIMO ANVC ${ }^{\star}$
}

\author{
J Luo* and S M Veres* \\ ${ }^{*}$ School of Engineering Sciences, University of Southampton, Highfield, \\ Southampton, SO17 1BJ, UK,
}

\begin{abstract}
A new iterative feedback/feedforward tuning (IFFT) method is presented for multiple-input multiple output (MIMO) control systems that relies on efficient computation of the negative gradient of the controller cost function in the frequency domain. The iterative method is using only one experiment per iteration and it is therefore suitable for realtime implementation for periodic adjustment of the controller. The primary target application area of the presented method is self-tuning feedback control in active noise and vibration control (ANVC).
\end{abstract}

Keywords: self tuning control; iterative method; active noise/vibration control; frequency domain; multi-input/multi-output system

\section{INTRODUCTION}

Iterative feedback tuning (IFT) has been the subject of intensive research efforts during the past decade (Hjalmarsson and Gevers M, 1998; Hjalmarsson, 1999; Hjalmarsson, 2002). Its advantage is that it does not need modelling, given a stable and reasonably functioning controller to start with is available. Recent efforts were aimed at improving gradient estimation (Jansson et al., 2002; Jansson and Hjalmarsson, 2004) of the control performance criterion, improving stability robustness without modelling (Veres and Hjalmarsson, 2002) and through spectrum analysis (Kammer et al., 2000).

In (Luo and Veres, n.d.), Iterative Feedback/Feedforward Tuning (IFFT) in Frequency Domain(FD), i.e., FD-IFFT, has been proposed to solve SISO ANVC problems. This paper extends FD-IFFT to Multiple Input Multiple Output (MIMO) cases that relies on the frequency domain handling of signals and dynamics in multi-variable systems. It only requires one additional experiment for each input channel of the unknown plant to compute a gradient estimate. Tuning simultaneously takes place while the iterative feedback tuning (IFT) in earlier publications had to perform multiple experiments for feedback and feedforward controllers.

Although the new approach is applicable to general control problems in theory, it is particularly suitable for control problems with finite frequency spectrum signals (disturbances, references and measurements) in practice. The most important target area of its application is active noise/vibration control (ANVC) of low frequency sound and vibration that has been an active research field for almost three decades. The original idea of ANVC has been proposed in the early 1930s (Lueg, 1934), and the underlying physical theory has been laid down for some time (Tokhi and Veres, 2002). Many effective methods have been developed in the last twenty years. There are design approaches that are model-based, e.g., $H_{\infty}$ control (Elliott and Rafaely, 1997), unfalsified-model based control (Veres and Wall, 2000) and iterative correlation based IFT (Karimi et

\footnotetext{
^ Corresponding author S.M.Veres.Email: s.m.veres@ soton.ac.uk.
}

al., 2003). There are many model-free approaches, which do not model the plant dynamics with precise models, but tune the controller parameters directly. One of the most widely used and well understood methods is filtered-x LMS algorithm (Morgan, 1980), which can be made adaptive to track plant dynamics with slow time variance. Frequency selective filter-based iterative feedback/feedforward tuning (FSF-IFFT) control (Meurers and Veres, 1999) has also been successfully used for periodic disturbance cancelation. There are very few effective methods available to tune both feedback/feedforward controllers in ANVC.

The remainder of this paper is organized as follows. In Section II, the problem of gradient-based tuning control for ANVC is shortly reviewed in the time domain. In Section III, the idea of FD-IFFT is proposed and some implementation topics are discussed. In Section IV, a series of MIMO simulation examples are presented to compare two implementations of FD-IFFT. Finally conclusions are drawn in the last section.

\section{GRADIENT BASED TUNING FOR ANVC}

In this section, the ANVC problem is generally addressed, the fundamental definitions and performance function will be defined.

Following symbols are frequently used in the paper. 


\begin{tabular}{|c||l|}
\hline \multicolumn{1}{|c||}{$\nabla$} & Gradient vector of functions \\
\hline$\mapsto$ & Map to \\
\hline$:=$ & Define or denote \\
\hline$\{\cdot\}^{T}$ & Transpose \\
\hline$\{\cdot\}^{*}$ & Conjugate and transpose \\
\hline$\phi_{\{\cdot\}}$ & Discrete spectrum of a signal \\
\hline$\phi_{\{\cdot\}} \mid \omega$ & $\begin{array}{l}\text { Discrete spectrum of a signal } \\
\text { over frequency subset } \omega\end{array}$ \\
\hline$\Phi_{\{\cdot\}}$ & $\begin{array}{l}\text { Discrete frequency response function } \\
\text { of a dynamics }\end{array}$ \\
\hline$\Phi_{\{\cdot\}} \mid \omega$ & $\begin{array}{l}\text { Discrete frequency response over } \\
\text { frequency subset } \omega\end{array}$ \\
\hline DFT & Discrete Fourier transform \\
\hline diag $(\boldsymbol{x})$ & Diagonal matrix with diagonal vector $\boldsymbol{x}$ \\
\hline FRF & Frequency Response Function \\
\hline LTI & Linear Time Invariant System \\
\hline
\end{tabular}

Fig. 1 gives a schematic description of the control system considered.

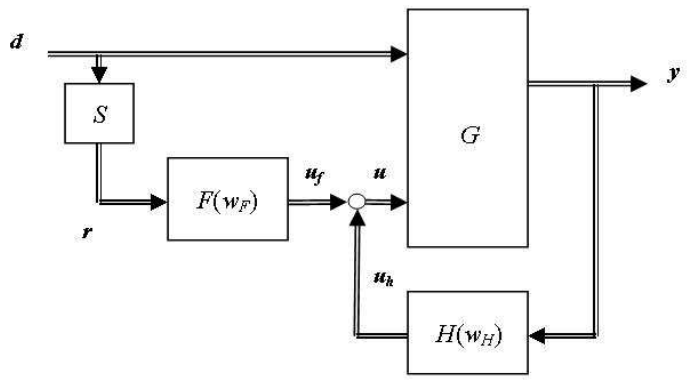

Fig. 1. Block diagram of a linear feedforward feedback system of ANVC system

The measured output, which is affected by the disturbance $\boldsymbol{d} \in \mathbb{R}^{n_{d}}$, is represented by $\boldsymbol{y} \in \mathbb{R}^{n_{y}}$. $G$ is the unknown plant dynamics with inputs $\boldsymbol{d}$ and $\boldsymbol{u}$ and produce $\boldsymbol{y}$. It can be described as

$$
\boldsymbol{y}=G(\boldsymbol{d}, \boldsymbol{u})
$$

The control signals from the feedforward controller $F$ and feedback controller $H$ are denoted by $\boldsymbol{u}_{f} \in \mathbb{R}^{n_{u}}$ and $\boldsymbol{u}_{h} \in$ $\mathbb{R}^{n_{u}}$, respectively. The tunable control system $C$ comprises the parameterized feedforward controller $F$ and the feedback controller $H$ :

$$
C(\boldsymbol{w}, \boldsymbol{r}, \boldsymbol{y}): \quad \begin{aligned}
F: \boldsymbol{u}_{f} & =F\left(\boldsymbol{w}_{F}, \boldsymbol{r}\right) \\
H: \boldsymbol{u}_{h} & =H\left(\boldsymbol{w}_{H}, \boldsymbol{y}\right) \\
\boldsymbol{u} & =\boldsymbol{u}_{f}+\boldsymbol{u}_{h}
\end{aligned}
$$

which can be tuned by adjusting their parameter vectors in $\boldsymbol{w}:=\left\{\boldsymbol{w}_{F}, \boldsymbol{w}_{H}\right\} \in \mathbb{R}^{n_{w}}$.

The disturbance-reference signal $\boldsymbol{r} \in \mathbb{R}^{n_{r}}$ is obtained through an unknown but time-invariant dynamics $S$ from $\boldsymbol{d}$. While the output signal $\boldsymbol{y}(t)$ is measurable and recordable, the disturbance signal $\boldsymbol{d}$ cannot be measured directly.

In case of periodic disturbance $\boldsymbol{d}$, it is always assumed that the steady output $y$ is also periodic. If the ANVC system has steady output $\boldsymbol{y}$ with period $N$ then the control performance criterion is defined as the average quadratic performance of a length $N$ output sequence:

$$
J(w):=\frac{1}{N} \sum_{t=0}^{N-1} y^{\mathrm{T}}(t) Q y(t)
$$

where $Q$ is a priori known weighting matrix.

The objective of tuning for ANVC is to adjust the controller parameters $\boldsymbol{w}$ to minimize performance (3).

\section{ITERATIVE TUNING IN THE FREQUENCY DOMAIN}

In this section a general framework of frequency domain iterative feedback-feedforward tuning (FD-IFFT) is introduced and some implementation issues are also discussed.

\subsection{Gradient estimate in the frequency domain}

Considering the MIMO system described by Fig.1, if there is $N$-length output data set $\mathscr{Y}:=\{\boldsymbol{y}(0) ; \ldots ; \boldsymbol{y}(N-1)\}, \boldsymbol{y}(t):=$ $\left\{y_{1}(t), \ldots, y_{n y}(t)\right\} \in \mathrm{R}^{n_{y}}$, it can be rewritten with the order of output channels as $\mathscr{Y}=\left\{\boldsymbol{y}_{1}, \ldots, \boldsymbol{y}_{n_{y}}\right\}, \boldsymbol{y}_{i}=\left\{y_{i}(0) ; \ldots ; y_{i}(N-\right.$ $1)\}, i=1, \ldots, n_{y}$.

Denoting $\omega_{m}:=\frac{2 \pi}{N} m, m=0, \ldots, N-1$ as $m$-th discrete frequency for $N$-length data, $\boldsymbol{\phi}_{y}^{i}:=\left\{\phi_{y}^{i}\left(\omega_{0}\right) ; \ldots ; \phi_{y}^{i}\left(\omega_{N-1}\right)\right\} \in \mathbb{C}^{N}$ denotes the discrete spectrum of $N$-length $\boldsymbol{y}_{i}$, which can be estimated by $\boldsymbol{\phi}_{y}^{i} \doteq \operatorname{DFT}\left(\boldsymbol{y}_{i}\right)$. Furthermore, the discrete spectrum of $\mathscr{Y}$ is described as $\boldsymbol{\phi}_{y}:=\left\{\boldsymbol{\phi}_{y}^{1} ; \ldots ; \boldsymbol{\phi}_{y}^{n_{y}}\right\} \in \mathbb{C}^{\left(n_{y} \times N\right) \times 1}$. There are similar notations as used such as $\boldsymbol{\phi}_{d}, \boldsymbol{\phi}_{r}, \boldsymbol{\phi}_{u f}$ and $\boldsymbol{\phi}_{u h}$.

In the frequency domain, the plant $G$ is described as function $\left\{\boldsymbol{\phi}_{d}, \boldsymbol{\phi}_{u}\right\} \mapsto \boldsymbol{\phi}_{y}$ :

$$
\boldsymbol{\phi}_{y}=\Phi_{G}\left(\boldsymbol{\phi}_{d}, \boldsymbol{\phi}_{u}\right)=\Phi_{G}\left(\boldsymbol{\phi}_{d}, \boldsymbol{\phi}_{u}^{1}, \ldots, \phi_{u}^{n_{u}}\right)
$$

and controller system $C$ is described as function $\left\{\boldsymbol{w}, \boldsymbol{\phi}_{r}, \boldsymbol{\phi}_{y}\right\} \mapsto$ $\phi_{u}$ :

$$
\begin{array}{ll}
\Phi_{C}\left(\boldsymbol{w}, \boldsymbol{\phi}_{r}, \boldsymbol{\phi}_{y}\right): & \Phi_{F}: \boldsymbol{\phi}_{u f}=\Phi_{F}\left(\boldsymbol{w}_{F}, \boldsymbol{\phi}_{r}\right) \\
& \Phi_{H}: \boldsymbol{\phi}_{u h}=\Phi_{H}\left(\boldsymbol{w}_{H}, \boldsymbol{\phi}_{y}\right)
\end{array}
$$

In LTI systems, FRF $\Phi_{G}, \Phi_{H}$ and $\Phi_{F}$ are derivative functions with respect to inputs' spectrum.

Therefore, some notations can be defined as follows: $\Phi_{G}:=$ $\frac{\partial \boldsymbol{\phi}_{y}}{\partial \boldsymbol{\phi}_{u}} \in \mathbb{C}^{\left(n_{y} \times N\right) \times\left(n_{u} \times N\right)}, \Phi_{F}:=\frac{\partial \boldsymbol{\phi}_{u f}}{\partial \boldsymbol{\phi}_{r}} \in \mathbb{C}^{\left(n_{u} \times N\right) \times\left(n_{r} \times N\right)}, \Phi_{F}^{(w, u)}:=$ $\frac{\partial \boldsymbol{\phi}_{u f}}{\partial \boldsymbol{w}_{F}} \in \mathbb{C}^{\left(n_{u} \times N\right) \times\left(n_{w f}\right)}, \Phi_{H}:=\frac{\partial \boldsymbol{\phi}_{u h}}{\partial \boldsymbol{\phi}_{y}} \in \mathbb{C}^{\left(n_{u} \times N\right) \times\left(n_{y} \times N\right)}$ and $\Phi_{H}^{(w, u)}:=$ $\frac{\partial \boldsymbol{\phi}_{u h}}{\partial \boldsymbol{w}_{H}} \in \mathbb{C}^{\left(n_{u} \times N\right) \times\left(n_{w h}\right)}$.

Considering LTI in Fig. 1, the plant $G$ in frequency domain can be written with increment format as

$$
\Delta \phi_{y}=\Phi_{G}\left(\Delta \phi_{u f}+\Delta \phi_{u h}\right)
$$

With regard to the small increment of parameter $\boldsymbol{w}$, i.e., $\Delta \boldsymbol{w}_{F}$ and $\Delta \boldsymbol{w}_{H}$, it is straight forward to write

$$
\Delta \boldsymbol{\phi}_{y}=\Phi_{G}\left(\Phi_{F}^{(w, u)} \Delta \boldsymbol{w}_{F}+\Phi_{H}^{(w, u)} \Delta \boldsymbol{w}_{H}+\Phi_{H} \Delta \boldsymbol{\phi}_{y}\right)
$$

Using notations $\Delta \boldsymbol{\phi}_{u f}^{w}:=\Phi_{F}^{(w, u)} \Delta \boldsymbol{w}_{F}, \Delta \boldsymbol{\phi}_{u h}^{w}:=\Phi_{H}^{(w, u)} \Delta \boldsymbol{w}_{H}$ and $\Delta \phi_{u h}^{y}:=\Phi_{H} \Delta \phi_{y}$, the incremental relationship (7) can be graphically described by following Fig 2 . 


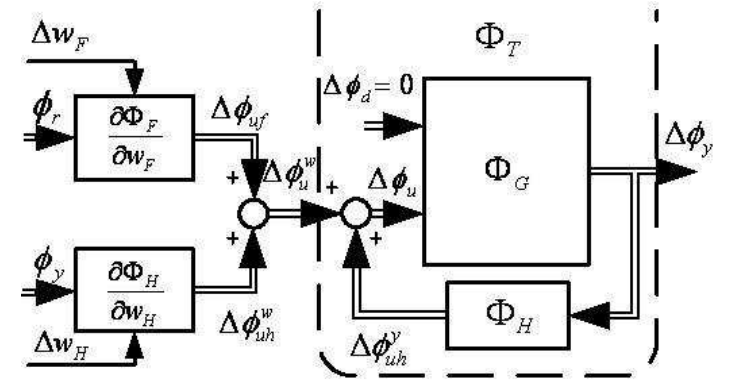

Fig. 2. Block diagram of small increment in frequency domain

If $\left(I-\Phi_{G} \Phi_{H}\right)^{-1}$ exists, the input/output mapping $\Delta \boldsymbol{\phi}_{u}^{w} \mapsto \Delta \boldsymbol{\phi}_{y}$ can be rewritten from (7) as

$$
\Delta \phi_{y}=\left(I-\Phi_{G} \Phi_{H}\right)^{-1} \Phi_{G}\left(\Delta \phi_{u f}^{w}+\Delta \phi_{u h}^{w}\right)
$$

Considering LTI closed loop dynamics $T:=\{G, H\}$, the FRF of $T$ is defined as

$$
\Phi_{T}:=\left(I-\Phi_{G} \Phi_{H}\right)^{-1} \Phi_{G} \in \mathbb{C}^{\left(n_{y} \times N\right) \times\left(n_{u} \times N\right)},
$$

From (8), the derivative of $\boldsymbol{\phi}_{y}$ with respect to controller parameters $\boldsymbol{w}_{H}$ and $\boldsymbol{w}_{F}$ can be written as

$$
\begin{aligned}
\frac{\partial \boldsymbol{\phi}_{y}}{\partial \boldsymbol{w}_{H}} & =\Phi_{T} \Phi_{H}^{(w, u)} \\
\frac{\partial \boldsymbol{\phi}_{y}}{\partial \boldsymbol{w}_{F}} & =\Phi_{T} \Phi_{F}^{(w, u)}
\end{aligned}
$$

According to Parseval's theorem (Oppenheim and Willsky, 1996), it is straightforward to write (3) in the frequency domain format as

$$
J=\frac{1}{N^{2}} \sum_{i=1}^{n_{y}} \sum_{j=0}^{N-1} \phi_{y}^{i *}\left(\omega_{j}\right) q_{i} \phi_{y}^{i}\left(\omega_{j}\right)=\frac{1}{N^{2}} \boldsymbol{\phi}_{y}^{*} Q_{F} \boldsymbol{\phi}_{y}
$$

where $Q_{F} \in \mathbb{R}^{\left(n_{y} \times N\right) \times\left(n_{y} \times N\right)}$ is the performance weighting matrix $Q$ in the frequency domain.

The derivative of performance $J$ with respect to controller parameters can be written as

$$
\frac{\partial J(\boldsymbol{w})}{\partial w_{i}}=\frac{2}{N^{2}} \boldsymbol{\phi}_{y}^{*} Q_{F} \Phi_{T} \Phi_{C}^{\left(w_{i}, u\right)}
$$

where $\Phi_{C}^{\left(w_{i}, u\right)}:=\frac{\partial \Phi_{C}\left(\boldsymbol{w}, \boldsymbol{\phi}_{y}, \boldsymbol{\phi}_{r}\right)}{\partial \boldsymbol{w}_{i}}$.

\subsection{Tuning of MIMO systems in the frequency domain}

In (13) the key to estimate $\frac{\partial J(w)}{\partial w_{i}}$ is to compute $\Phi_{T}$, which has $n_{y} \times N$ rows and $n_{u} \times N$ columns.

In this subsection, some assumptions are introduced and some estimation techniques are given to simplify the estimation of $\Phi_{T}$, and the practical tuning strategy is proposed for the MIMO ANVC problem.

First note that most disturbance signals in engineering can often be considered to have finite discrete spectrum, especially in ANVC problems. For a periodic output $\boldsymbol{y}$ with common period $N$, only a finite set of frequencies, $\boldsymbol{\omega}=\left\{\omega_{1}, \ldots, \omega_{n_{\omega}}\right\}$, are included in $\phi_{y}$, the other elements in $\phi_{y}$ are 0 . In this case, (9) can be written as finite frequency format as

$$
\left.\Phi_{T}\right|_{\omega}=\left.\left(\boldsymbol{I}-\left.\left.\Phi_{G}\right|_{\omega} \Phi_{H}^{i}\right|_{\omega}\right)^{-1} \Phi_{G}\right|_{\omega}
$$

and similarly (13) can be rewritten as

$$
\nabla J\left(\boldsymbol{w}^{i}\right)=\left.\left.\left.\frac{2}{N^{2}} \boldsymbol{\phi}_{y}^{*}\right|_{\omega} \Phi_{T}^{i}\right|_{\omega} \Phi_{C}^{(w, u)}\right|_{\omega}
$$

Remark 1. It should be noted that, in the previous subsection, there was no limitation about the spectrum of $\boldsymbol{y}, \boldsymbol{r}$ and $\boldsymbol{u}$. Theoretically, the gradient based tuning described by (10), (11) and (13) is applicable for any LTI control problems. There is similar idea arised in (Kammer et al., 2000) for servo control.

Secondly, an indirect estimate of $\Phi_{T}$ is more convenient for the online tuning. According to (9), if $\Phi_{G}$ can be estimated, $\Phi_{T}$ can be solved since $H$ is known by the designer.

Note that if $\Phi_{G}$ is assumed a LTI system, FRF is independent with respect to different frequencies. To ease the notation, for a single frequency FRF of $\Phi_{G}$, the $\Phi_{G}(\omega)$ is used in the following discussion, and the extension to complete $\Phi_{G}$ is straightforward.

Note that $\Phi_{G}(\omega) \in \mathbb{C}^{n_{y} \times n_{u}}$ has $n_{y} \times n_{u}$ unknown variables, which can be solved out through a full-rank $n_{y} \times n_{u}$ equation matrix.

Considering plant $G$, it is straightforward to get the an equation system

$$
\Delta \phi_{y}(\omega)=\Phi_{G}(\omega) \Delta \phi_{u}(\omega)
$$

which gives $n_{y}$ equations.

Therefore, considering the case of the full rank equations, given $n_{u}$ such equation groups as in (16), $\Phi_{G}(\omega)$ can be obtained by solving an equation system with $n_{u} \times n_{y}$ equations.

To summarize, under the assumption of a finite frequency set $\boldsymbol{\omega}$ for the disturbance and assuming an LTI system, we have following tuning strategy in the frequency domain:

At the $i$-th iteration,

(1) Estimate $\left.\Phi_{G}\right|_{\omega}$ by solving the equation set from (16);

(2) Calculate $\left.\Phi_{T}^{i}\right|_{\omega}$ with (14);

(3) Solve the derivative of $J$ with (15);

(4) Update the controller parameter $\boldsymbol{w}$ with

$$
\boldsymbol{w}^{i+1}=\boldsymbol{w}^{i}-\mu \nabla J\left(\boldsymbol{w}^{i}\right)
$$

where $\mu$ is a proper step size to update the controller.

Remark 2. As above stated, at least $n_{u}$ different equation groups as (14) are required to solve $\Phi_{G}(\omega)$, which means $n_{u}$ pairs of difference data $\{\Delta \boldsymbol{u}, \Delta \boldsymbol{y}\}$ are required. In the implementation, in order to get the estimate of $\left.\Phi_{G}\right|_{\omega}, 1+n_{u}$ experiments are required to yield $n_{u}$ pairs of $\{\Delta \boldsymbol{u}, \Delta \boldsymbol{y}\}$.

For LTI systems, $\left.\Phi_{G}\right|_{\omega}$ is considered unchanged and can be estimated offline. $\left.\Phi_{T}\right|_{\omega}\left(\Phi_{G}\left|\omega, \Phi_{H}^{i}\right|_{\omega}\right)$ can be updated with the change of $H^{i}$. Therefore, to make $N_{T}$ times gradient based tuning, plus the $n_{u}$ additional experiments, $N_{T}+n_{u}$ time iterations are necessary to be performed. 


\subsection{Further discussion about the implementation}

In this subsection some issues are discussed with regard to the implementation of FD-IFFT in practice.

First note that $N$ should be the common period of $\boldsymbol{y}$ and $\boldsymbol{r}$. In the time domain, (3) vary with the starting point if $N$ is not the common period of $\boldsymbol{y}$. In the frequency domain, while $\boldsymbol{\phi}_{y}$ and $\boldsymbol{\phi}_{r}$ is the discrete spectrum based on $N$ length time series data, the necessary condition to make $\boldsymbol{\phi}_{y}=\operatorname{DFT}(\boldsymbol{y})$ and $\boldsymbol{\phi}_{r}=\operatorname{DFT}(\boldsymbol{r})$ is that $\boldsymbol{y}$ and $\boldsymbol{r}$ has common period of $N$. In the practice, the common period $N$ is often offline estimated, otherwise requires a proper long period experiment to make online estimate.

Secondly, the format of tunable $C=\{F, H\}$ can vary in different applications.

It is noted that there is no limitation about the format of the controllers in (15).

FIR controllers are one of the most convenient controller structures to realize. For some simple ANVC applications with few frequencies, especially single frequency control, FIR controllers can give the satisfying tuning result with the simple controller structure and easy tuning algorithm, which is important in practice.

In order to achieve higher level of cancelation performance and more robust tuning performance, the Frequency-SelectiveFilter (FSF) can be introduced for each of main frequencies in the signal, which is called FSF-FD-IFFT in the following discussion.

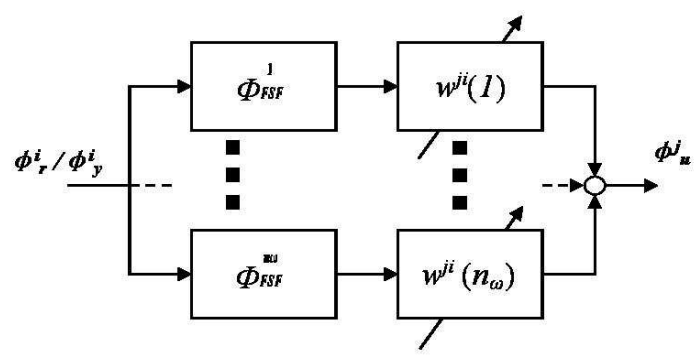

Fig. 3. Block diagram in frequency domain of one sub-block in the FSF controller

Fig. 3 shows the typical block diagram of one sub-block in the FSF controller with $n_{\omega}$ FSF channels, which is from the $i$-th input to the $j$-th output. For the finite set of $\boldsymbol{\omega}=\left\{\omega_{1}, \ldots, \omega_{n_{\omega}}\right\}$, there are $n_{\omega}$ FSF channels. $\Phi_{F S F}^{m}$ denotes the band-pass FSF with central frequency $\omega_{m}$, which cascaded with a tunable complex gain module $w^{j i}(m)$, where $m=1, \ldots, n_{\omega}$.

\section{SIMULATION}

This section illustrates the usefulness of the FD-IFFT as tested in simulation using MATLAB. FIR control structure and FSFFD-IFFT are tested and compared. The robustness against the error in the common period $N$ is discussed with a simulation example.

\subsection{Simulation platform}

The block diagram of the Simulink-based simulation is given in Fig. 4. It is a 2-input and 2-output LTI system. $y 1, y 2, r 1$ and $r 2$ denote the data acquired for output and reference signals.
Module $N y 1, N y 2, N r 1$ and $N r 2$ denote the senor noise in the output and reference paths. They are assumed as white noise with standard deviation 0.001 .

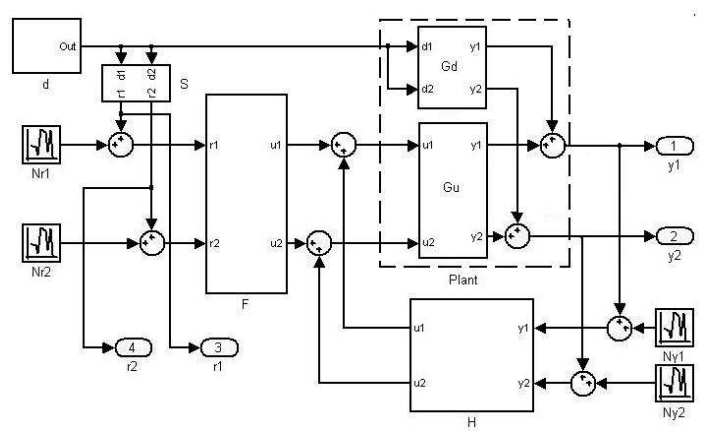

Fig. 4. Block diagram for simulation

In Fig. 4, control path $G_{u}$ is given by

$$
\left[\begin{array}{cc}
\frac{0.1 q^{-8}-0.3 q^{-9}}{1+0.2 q^{-1}-0.2 q^{-2}} & \frac{0.01 q^{-6}-0.03 q^{-7}}{1+0.02 q^{-1}-0.02 q^{-2}} \\
\frac{-0.02 q^{-7}-0.02 q^{-8}}{1+0.01 q^{-1}-0.01 q^{-2}} & \frac{-0.2 q^{-8}-0.3 q^{-9}}{1+0.1 q^{-1}-0.2 q^{-2}}
\end{array}\right]
$$

and disturbance path $G_{d}$ is given by

$$
\left[\begin{array}{cc}
\frac{0.85 q^{-4}}{1+0.4 q^{-1}} & 0 \\
0 & \frac{0.95 q^{-6}}{1-0.2 q^{-2}}
\end{array}\right]
$$

The sampling frequency is $4 \mathrm{kHz}$. The disturbance signal $\boldsymbol{d}$ is a mix of three sine-waves with frequencies of $50 \mathrm{~Hz}, 80 \mathrm{~Hz}$ and $100 \mathrm{~Hz}$ and a white noise signal $w_{t}$ with standard deviation 0.01 , leading to:

$$
\begin{aligned}
d(t)= & \frac{1}{3}[\sin (100 \pi t)+\sin (160 \pi(t-0.091)) \\
& +\sin (200 \pi t)]+w_{t}
\end{aligned}
$$

The uncontrolled output is shown in Fig. 5.
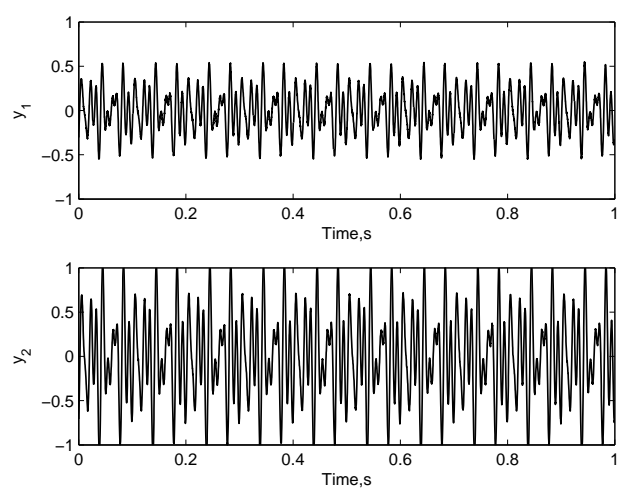

Fig. 5. Initial output without control

The reference signal $r(t)$ is obtained from $d(t)$ by $S$ : 


$$
S(q)=\left[\begin{array}{cc}
\frac{0.8 q^{-8}}{1+0.8 q^{-1}} & 0 \\
0 & \frac{0.5 q^{-10}}{1+0.9 q^{-1}}
\end{array}\right]
$$

\subsection{Simulation for FIR-FD-IFFT and FSF-FD-IFFT}

In this subsection, two formats of the controller structure are tested with the above simulated platform. One is an FIR controller structure (FIR-FD-IFFT), another is FSF-FD-IFFT.

FIR-FD-IFFT has the 10-th order feedback controller and the 40-th order feedforward controller. The step size (adaptation gain) for feedforward controller tuning is $\mu_{f}=0.1$ and step size for feedback controller tuning is $\mu_{h}=0.02$.

In FSF-FD-IFFT, 1st-order Butterworth bandpass filters are online designed according to the spectrum of $y$. The bandwidths of the FSF were given by the disturbance frequency \pm 10 percent which also eliminates the unwanted white noise in the tuning. The step size (adaptation gain) for feedforward controller tuning is $\mu_{f}=8.0$ and step size for feedback controller tuning is $\mu_{h}=2.0$.

The signal period is defined as $N=800$. The iteration time of tuning is set 50. All the initial controllers are set to zeros. The weighting matrix is $Q=\operatorname{diag}([1.00 .8])$. The initial performance criterion without control is 0.2443 . In order to perform an initial estimate of $G$, only the sub-block from $\boldsymbol{r}_{1}$ to $\boldsymbol{u}_{f 1}$ in $H$ is changed to be 0.2 in the 2 nd iteration, and only the sub-block from $\boldsymbol{r}_{2}$ to $\boldsymbol{u}_{f 2}$ in $H$ is changed to be 0.2 in the 3 rd iteration.

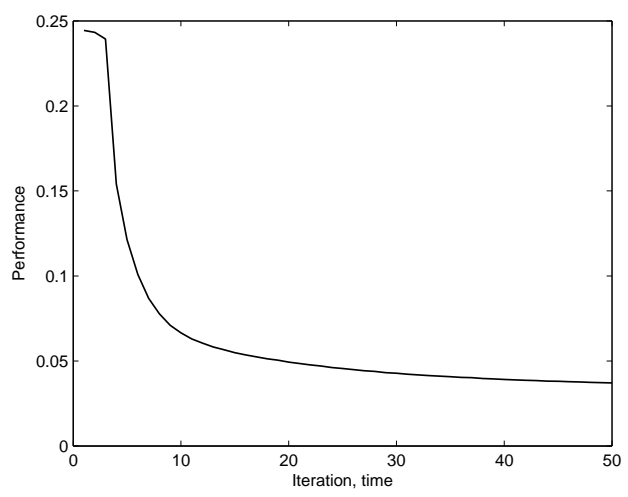

Fig. 6. Performance update in FIR-FD-IFFT
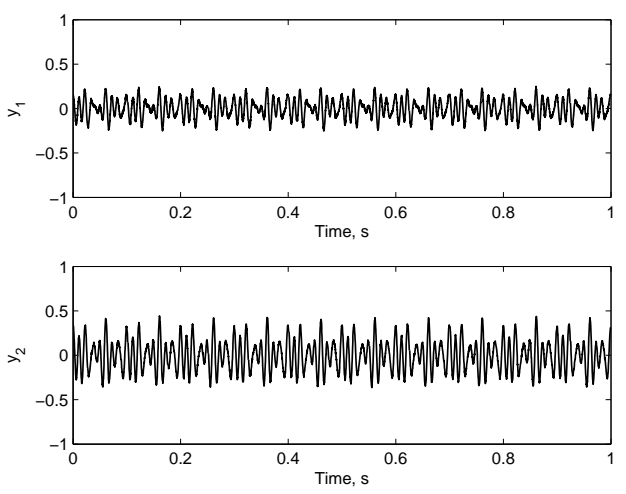

Fig. 7. Final output of FIR-FD-IFFT
Fig. 6 shows the updating performance in FIR-FD-IFFT: The 2nd and 3rd iteration are manual updates, which give $J(2)=$ 0.2432 and $J(2)=0.2391$. After 50 iterations, the final performance is $J=0.0371$ with $8.2 \mathrm{~dB}$ cancelation. The final output with control is shown in Fig.7.

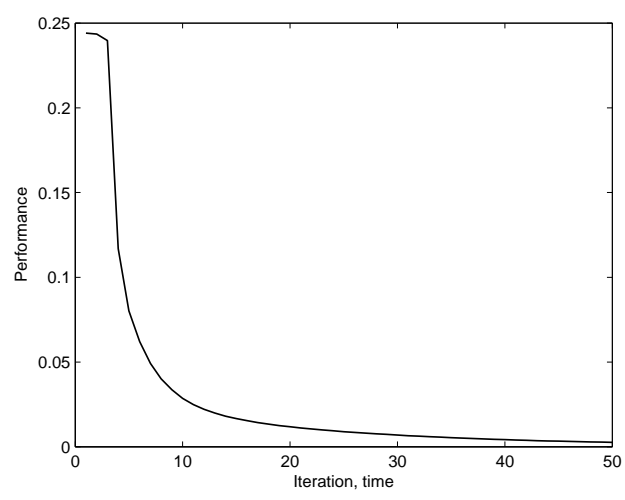

Fig. 8. Performance update in FSF-FD-IFFT
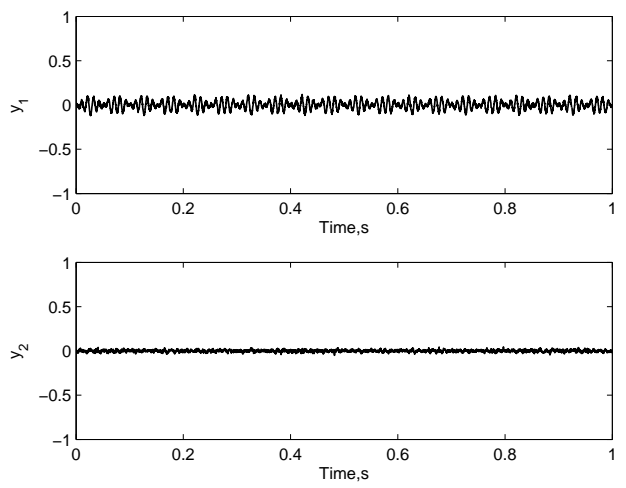

Fig. 9. Final output of FSF-FD-IFFT

Fig. 8 shows the updating performance in FSF-FD-IFFT. After 50 iterations, the final performance is $J=0.0026$ with $19.8 \mathrm{~dB}$ cancelation. The final output with control is shown in Fig.9.

It is obvious that FSF-FD-IFFT can supply much better tuning performance than FIR-FD-IFFT.

\section{EPILOGUE}

A hybrid iterative feedback/feedforward tuning approach in the frequency domain has been presented that uses an innovative way of computing gradient estimates of the controller cost function. Compared to IFT in the time domain, this method simplifies both control structure and control operation.

The method is ideally suitable for ANVC applications with feedback and feedforward controllers. After providing a general general idea about iterative tuning in the frequency domain, some detail issues were discussed for applications. The effectiveness and flexibility of FD-IFFT was shown by simulation examples.

As the basic scheme was outlined and tested in simulation, the robustness of controller is still questionable. Future work on robustification will be possible to perform directly in the frequency domain. Extension of the general framework to other control application except ANVC also requires further research. 


\section{REFERENCES}

Elliott, S. J. and B. Rafaely (1997). Frequency-domain adaptation of feedforward and feedback controllers. In: Proc. ACTIVE'97. pp. 75-92.

Hjalmarsson, H. (1999). Efficient tuning of linear multivariable controllers using iterative feedback tuning. Int. J. Adaptive Control and Signal Processing 13, 553-572.

Hjalmarsson, H. (2002). Iterative feedback tuning - an overview. Int. J. Adaptive Control and Signal Processing 16, 373 - 395.

Hjalmarsson, H. and Lequin O. Gevers M (1998). Iterative feedback tuning: theory and applications. IEEE Control Systems Magazine 18(4), 26-41.

Jansson, H. and H. Hjalmarsson (2004). Gradient approximations in iterative feedback tuning for multivariable processes. Int. J. Adaptive Control and Signal Processing 18(8), 665-681.

Jansson, H., H. Hjalmarsson and A. Hansson (2002). On methods for gradient estimation in IFT for MIMO systems. In: 15th World Congress on Automatic Control.

Kammer, L. C., R. R. Bitmead and P. L. Bartlett (2000). Direct iterative tuning via spectral analysis. Automatica 36(9), 1301-1307.

Karimi, A., L. Mikovi and D. Bonvin (2003). Iterative correlation-based controller tuning: application to a magnetic suspension system. Control Engineering Practice 11, 1069-1087.

Lueg, P. (1934). Process of silencing sound oscillations, U.S. pantent 043,416.

Luo, Jian and S. M. Veres (n.d.). Iterative feedback/feedfoward tuning control in the frequency domain for AVNC. In: ECC'07. Acceptted.

Meurers, T. and S. M. Veres (1999). Iterative design for vibration attenuation. Int. J. Acoustics and Vibration 4(2), 7983.

Morgan, D. R. (1980). An analysisi of multiple correlation cancellation loops with a filter in the auxiliary path. IEEE Trans. on Acoustics, Speech and Signal Processing 28(4), 454-467.

Oppenheim, A. V. and A. S. Willsky (1996). Signals and Systems. 2nd ed.. Prentice Hall.

Tokhi, O. and Veres, S. M., Eds. (2002). Active sound and vibration control: theory and applications. IEE.

Veres, S. M. and D. S. Wall (2000). Synergy and duality of identification and control. Taylor \& Francis, London.

Veres, S. M. and H. Hjalmarsson (2002). Tuning for robustness and performance using iterative feedback tuning. 42nd IEEE Conference on Decision and Control 4, 4682 - 4687. 